Cahiers $d u$ MONDE RUSSE

\section{Cahiers du monde russe}

Russie - Empire russe - Union soviétique et États indépendants

$51 / 4 \mid 2010$

Sciences humaines et sociales en Russie à l'Âge d'argent

\title{
A.Ju. Volodin, Istorija fabričnoj inspekcii 1882-1914
}

Jean-Paul Depretto

\section{(2) OpenEdition}

Journals

Édition électronique

URL : https://journals.openedition.org/monderusse/7374

DOI : 10.4000/monderusse. 7374

ISSN : $1777-5388$

Éditeur

Éditions de l'EHESS

Édition imprimée

Date de publication : 25 novembre 2010

Pagination : 688-691

ISBN : 978-2-7132-2316-7

ISSN : $1252-6576$

Référence électronique

Jean-Paul Depretto, «A.Ju. Volodin, Istorija fabričnoj inspekcii 1882-1914 », Cahiers du monde russe [En ligne], 51/4 | 2010, mis en ligne le 09 décembre 2011, consulté le 03 septembre 2022. URL : http:// journals.openedition.org/monderusse/7374; DOI : https://doi.org/10.4000/monderusse.7374

Ce document a été généré automatiquement le 3 septembre 2022

Tous droits réservés 


\title{
A.Ju. Volodin, Istorija fabričnoj inspekcii 1882-1914
}

\author{
Jean-Paul Depretto
}

\section{RÉFÉRENCE}

A.Ju. VoLoDIN, Istorija fabričnoj inspekcii 1882-1914 [Histoire de l'Inspection des

fabriques]. Moscou : Rosspen, 2009, $207 \mathrm{p}$.

1 Pour qui s'intéresse à l'Empire russe finissant, A.Ju. Volodin n'est pas un inconnu : il a déjà publié plusieurs articles sur l'Inspection des fabriques ${ }^{1}$. La présente étude comble une lacune dans l'historiographie russe, où n'existait pas de monographie comparable ; elle a été écrite dans le cadre d'un projet de recherche collectif, intitulé «L'Inspection des fabriques en Russie en tant qu'institution ", et s'appuie sur une thèse de troisième cycle soutenue en 2006 à l'université de Moscou. L'auteur se donne pour tâche d'étudier la mise en place institutionnelle de l'Inspection, créée en 1882, le développement et les transformations du corps des inspecteurs ainsi que leur fonction de médiation dans les conflits entre ouvriers et entrepreneurs. Disons d'emblée que Volodin porte un jugement très positif sur l'activité de ces inspecteurs : ils auraient été presque les seuls, selon lui, à respecter et à maintenir la légalité dans les rapports entre patrons et ouvriers.

2 L'approche pratiquée est à la fois institutionnelle et sociale: l'auteur se livre à un examen soigneux des textes juridiques qui régissent l'Inspection, tout en prêtant autant d'attention à la composition du personnel et à la description concrète de son activité. Il met l'accent sur trois fonctions de l'Inspection: contrôle de l'exécution des normes établies par la législation concernant les fabriques, réalisation d'enquêtes statistiques, et enfin médiation (déjà évoquée).

3 Volodin a mobilisé des sources nombreuses et variées: actes législatifs concernant l'Inspection; dossiers des administrations centrales dans le domaine du commerce et de l'industrie ; fonds d'un inspecteur de secteur de la province de Moscou, conservé aux Archives historiques centrales de Moscou; documents des commissions qui ont délibéré 
sur l'Inspection ; rapports des inspecteurs ; presse, y compris professionnelle ; Mémoires d'inspecteurs et d'hommes d'État. L'intérêt du livre est grandement rehaussé par la présence de riches annexes, où sont publiés d'abondants documents : les principaux actes législatifs sur l'Inspection des fabriques de 1882 à 1914, des exemples d'uniformes d'inspecteurs, une carte-plan de la ville industrielle de Serpuhov, deux tableaux statistiques concernant des plaintes d'ouvriers, une plainte manuscrite d'ouvrier, la réponse d'un industriel à un inspecteur du travail à propos d'une plainte.

Dans la conclusion (p.144-147), Volodin distingue trois périodes dans l'histoire de l'institution: création (1882-1893), réforme (1894-1904) et développement (1905-1914). Chacune de ces périodes fait l'objet d'un chapitre. Les débuts sont marqués par de grandes difficultés, puisqu'il faut régler la question des rapports avec la police, l'administration locale et le ministère ; de fait, il est difficile d'élargir la surveillance des fabriques, faute de moyens. En 1882, on ne compte que cinq postes d'inspecteurs; en 1884, malgré une augmentation du personnel, seules trois provinces font l'objet d'une surveillance: Moscou, Vladimir et Saint-Pétersbourg. La deuxième étape, liée à la période Witte, est celle de la réforme de l'Inspection, transformée en mécanisme réel de participation de l'État à la vie de la fabrique; on voit alors s'instaurer une surveillance stricte des mécontentements réciproques des ouvriers et des industriels. En 1894, Witte rattache l'Inspection au Département du commerce et de l'industrie du ministère des Finances, en accroît fortement les moyens humains et en étend la compétence géographique; finalement, en 1897, la surveillance des fabriques est élargie à toute la Russie d'Europe. En 1903, les inspecteurs locaux sont soumis, à titre provisoire, au pouvoir du gouverneur. L'Inspection est rattachée en 1905 au ministère du Commerce et de l'Industrie, créé le 27 octobre de la même année : le territoire soumis à sa surveillance est encore étendu et la pression des pouvoirs locaux sur l'institution, renforcée. Entre 1905 et 1914, les inspecteurs deviennent les promoteurs de la légalité dans les entreprises privées de Russie d'Europe, aussi bien sous la révolution de 1905 que dans les années suivantes où le développement industriel s'accélère.

Plutôt que de résumer les trois chapitres, arrêtons-nous sur la démarche de Volodin dans deux domaines : l'analyse micro-historique du $10^{e}$ secteur de l'Inspection de la province de Moscou (p. 79-115) et la prosopographie des inspecteurs au début du $\mathrm{xx}^{\mathrm{e}}$ siècle (p. 125-134), en commençant par ce second aspect. En 1905, l'Inspection est divisée en 231 secteurs, répartis dans 64 provinces réunies en six arrondissements; le nombre total d'inspecteurs est de 268 , chiffre qui semble très faible si l'on songe à l'étendue de l'Empire, aux difficultés de communication et à l'ampleur du travail effectué. Les provinces les mieux dotées sont celles de Moscou (23), Saint-Pétersbourg (18), Vladimir (12), Kiev (11) et Varsovie (11). Les inspecteurs ne sont pas des juristes, mais avant tout des techniciens spécialistes de l'usine, en particulier des machines: ingénieurstechniciens, ingénieurs-mécaniciens, techniciens, ingénieurs des mines et ingénieurs militaires, auxquels il faut ajouter cinq médecins. En général, la montée en grade dans le corps n'est pas liée au changement de province; les inspecteurs restent attachés à leur lieu d'exercice; enfin, l'ossature de l'institution est solide et durable. Volodin souligne que l'Inspection a attiré des gens éminents, qui ont laissé un bon souvenir comme acteurs et spécialistes de la politique industrielle russe, et retrace brièvement les parcours de cinq inspecteurs remarquables: P.A. Peskov, A.A. Mikulin, V.P. LitvinovFalinskij, A.N. Bykov et E.M. Dement'ev, ce dernier étant célèbre pour avoir écrit un 
livre sur la fabrique ${ }^{2}$. Toutes ces données font revivre les acteurs de l'Inspection et donnent chair à l'ouvrage.

6 Les Archives historiques centrales de Moscou (fonds 2005) ont permis d'étudier un aspect très important du travail quotidien des inspecteurs : l'examen des situations de conflit dans les entreprises industrielles. Le $10^{e}$ secteur du gouvernement de Moscou était chargé de la surveillance des entreprises de Serpuhov et de son uezd, soit 46 usines et près de 20000 ouvriers, le textile constituant ici la base de l'industrie. L'inspecteur avait trois activités principales: contrôle dans l'entreprise, accueil quotidien au secrétariat et correspondance avec les dirigeants des fabriques du secteur. Il était tenu d'étudier les plaintes, écrites et orales, des ouvriers et des patrons et de faire en sorte d'orienter les parties vers un accord pacifique; en l'absence d'accord, il devait les renvoyer au tribunal. La plainte était définie comme une déclaration concernant la violation de droits établis par la loi ; elle se distinguait de la demande, qui visait à la résolution de malentendus non régulés par la loi. Les ouvriers utilisaient très largement cette deuxième forme pour défendre leurs droits. Volodin a travaillé sur un ensemble de 137 plaintes, datant d'avril 1896 à novembre 1897 et de janvier à décembre 1900 : le thème le plus fréquent était le « licenciement injuste». En fait, les ouvriers ne voyaient pas de différence entre légal et juste: d'où des plaintes fréquentes contre l'injustice d'un licenciement légal. Les coups constituaient la deuxième cause de plainte, les pertes de salaire liées aux arrêts de la production représentant la troisième. Ces coups pouvaient être le fait d'un contremaître qui rossait un ouvrier, mais ils s'échangeaient aussi entre ouvriers. Ces derniers se plaignaient aussi des pots-de-vin exigés par le contremaître et ses aides, sous forme de boisson, et indispensables pour pouvoir travailler; mais pour l'inspecteur, ce phénomène était difficile à mettre en évidence. Ces plaintes nous offrent ainsi des aperçus sur le monde ouvrier d'autant plus précieux que les documents utilisés émanent des intéressés. Elles témoignent d'une forme de conscience collective : la majorité des plaintes anonymes commence par des formules comme "nous ouvriers", "nous tisserands "... Les ouvriers commencent à se percevoir comme une force.

7 Tout au long de son ouvrage, A.Ju. Volodin cite très largement ses sources, donnant généreusement la parole aux inspecteurs et aux autres acteurs. Ce n'est pas le moindre mérite d'une monographie qui a su dépasser une approche étroite de son sujet et utiliser l'Inspection des fabriques comme un observatoire permettant d'offrir une vision renouvelée de l'histoire industrielle russe. Ce volume confirme l'excellente qualité de la collection d'histoire économique de la maison d'édition Rosspen.

Otečestvennaja IstoriiaEkonomičeskaja Istoriia : ObozrenieFabrika, čto ona daët naseleniju i čto ona u nego berët 